\title{
Mathematical Modeling of Electrical Conductivity of Anisotropic Nanocomposite with Periodic Structure
}

\author{
Sergey Korchagin ${ }^{1} @$, Ekaterina Pleshakova ${ }^{1}$, Irina Alexandrova ${ }^{1}$, Vitaliy Dolgov ${ }^{1}$, Elena Dogadina ${ }^{1, *}$, \\ Denis Serdechnyy ${ }^{2, *}$ and Konstantin Bublikov ${ }^{3}$ \\ 1 Department of Data Analysis and Machine Learning, Financial University under the Government of the \\ Russian Federation, Shcherbakovskaya, 38, 105187 Moscow, Russia; sakorchagin@fa.ru (S.K.); \\ pleshakova_es@mail.ru (E.P.); IAleksandrova@fa.ru (I.A.); VIDolgov@fa.ru (V.D.) \\ 2 Department of Innovation Management, State University of Management, Ryazansky Pr., 99, \\ 109542 Moscow, Russia \\ 3 Institute of Electrical Engineering, Slovak Academy of Sciences, Dubravska cesta 3484/9, \\ 84104 Bratislava, Slovakia; elecbubl@savba.sk \\ * Correspondence: EPDogadina@fa.ru (E.D.); dv_serdechnyj@guu.ru (D.S.)
}

check for updates

Citation: Korchagin, S.; Pleshakova, E.; Alexandrova, I.; Dolgov, V.; Dogadina, E.; Serdechnyy, D.;

Bublikov, K. Mathematical Modeling of Electrical Conductivity of Anisotropic Nanocomposite with Periodic Structure. Mathematics 2021, 9, 2948. https://doi.org/10.3390/ math9222948

Academic Editor: Krzysztof Kamil Żur

Received: 21 October 2021

Accepted: 16 November 2021

Published: 18 November 2021

Publisher's Note: MDPI stays neutral with regard to jurisdictional claims in published maps and institutional affiliations.

Copyright: (c) 2021 by the authors. Licensee MDPI, Basel, Switzerland. This article is an open access article distributed under the terms and conditions of the Creative Commons Attribution (CC BY) license (https:/ / creativecommons.org/licenses/by/ $4.0 /)$.

\begin{abstract}
Composite materials consisting of a dielectric matrix with conductive inclusions are promising in the field of micro- and optoelectronics. The properties of a nanocomposite material are strongly influenced by the characteristics of the substances included in its composition, as well as the shape and size of inclusions and the orientation of particles in the matrix. The use of nanocomposite material has significantly expanded and covers various systems. The anisotropic form of inclusions is the main reason for the appearance of optical anisotropy. In this article, models and methods describing the electrical conductivity of a layered nanocomposite of a self-similar structure are proposed. The method of modeling the electrical conductivity of individual blocks, layers, and composite as a whole is carried out similarly to the method of determining the dielectric constant. The advantage of the method proposed in this paper is the removal of restrictions imposed on the theory of generalized conductivity associated with the need to set the dielectric constant.
\end{abstract}

Keywords: composite materials; mathematical modeling; electrical conductivity; layered nanocomposites; periodic structure

\section{Introduction}

Lately, there has been a rapid growth of research related to the synthesis of new materials with a hierarchical structure and individual properties. The process of designing and developing these new materials is becoming increasingly expensive and complex, and the use of known materials with the required properties for the formation of nanocomposites is becoming an increasingly accessible way for new opportunities. There is a wide range of innovative applications of nanocomposites, due to the fact that they have significantly improved properties due to the hardening of nanoparticles. Further use depends on the study of their structure, for this it is necessary to apply modeling to understand the behavior of nanocomposites. Nanocomposites are capturing new areas of application. Due to the fact that the requirements for materials are growing now, in particular, the need for light weight and high strength materials, nanocomposites are attractive for such industries as biomedical engineering, aerospace, and automotive. The study and identification of patterns of interaction of a high-frequency electromagnetic field with various media, including nanostructures, is a modern topical area of research. This is due to both the fundamental nature of the problem and the possibility of using nanocomposites in applied tasks (creation of radar elements and devices, synthesis of absorbing and selective materials, etc.). In order to get the right functionality to meet different applications, we need innovations on the frontiers of materials science, chemistry, physics, theory, and engineering that can facilitate 
the design and manufacture of materials. Practical realization of such tasks is possible only on the basis of a detailed understanding of the fundamental phenomena of the interaction of the electromagnetic field with matter: resonant bursts of dielectric permittivity; the Kerr effect; quantum-dimensional, surface effects.

Despite the abundance of works on this topic, there are practically no mathematical models of layered nanocomposites with a periodic structure. An important feature of nanocomposites with a periodic structure is that in some directions and scales it is necessary to take into account nanoscales, and in others-micro-dimensions. In such systems, the anisotropy of geometric and electrical characteristics cannot be neglected. Thus, the development of mathematical models and a software package for the study of the electrical properties of anisotropic nanocomposites with a periodic structure is an urgent task.

In this paper, models and methods describing the electrical conductivity of a layered nanocomposite of a self-similar structure are proposed. In [1], the authors explain the basic concepts of the theory of statistical percolation and percolation transfer, which are subsequently applied to specific experiments on heterogeneous ionic conductors. The method of modeling the electrical conductivity of individual blocks, layers and composite as a whole is carried out similarly to the method of determining the dielectric constant [2-4]. The work also presents a two-dimensional finite element model to study the effect of interphase zones on the general properties of the composite, the simulation results clearly show that the loss moduli of composites are either expanded or shifted, which corresponds to the absence or presence of a geometrically percolating interphase network [5].

To determine the electrical conductivity of blocks with a width of $0.5-8 \mathrm{~nm}$, where dimensional effects can be significant, a quantum mechanical [6-8] was used. Based on the Kubo-Greenwood equations, a model of the electrical conductivity of the block has been developed, taking into account the anisotropy of electrical and geometric properties and electron spin. The model assumes the presence of information about the electron energy of the normal and excited states, as well as about the filling numbers determined by the Fermi distribution for the normal and excited states. To obtain them, the method of electronic minimization was used. The Hartree-Fock method is used to obtain wave functions. To calculate the electrical conductivity of plane-parallel layers, including blocks with a width of $0.5-32 \mathrm{~nm}$, a model of an effective medium for electrical conductivity was used. To determine the electrical conductivity of plane-parallel layers, including blocks with a width of $64-256 \mathrm{~nm}$ and the composite as a whole, the method of equivalent substitution schemes was used. The results obtained using the method proposed in this paper for determining electrical conductivity are compared with the results obtained using the currently known theory of generalized conductivity [9-11]. The results obtained are in good agreement. The advantage of the method proposed in this paper is the removal of restrictions imposed on the theory of generalized conductivity associated with the need to set the dielectric constant.

\section{Materials and Methods}

2.1. Mathematical Modeling and Method for Determining the Electrical Conductivity of a Layered Nanocomposite with a Periodic Structure

The method of modeling the electrical conductivity of individual blocks, layers, and the composite as a whole (for example, a nanocomposite consisting of 10 layers, see Table 1 ) is carried out analogously to the method of determining the dielectric permittivity [12-14] and is divided into 3 stages:

(1) to determine the electrical conductivity of blocks with a width of $0.5 \mathrm{~nm}$ (10th layer), $1 \mathrm{~nm}$ (9th layer), $2 \mathrm{~nm}$ (8th layer), $4 \mathrm{~nm}$ (7th layer), $8 \mathrm{~nm}$ (6th layer), where dimensional effects can be significant, a quantum mechanical calculation method (Kubo-Greenwood approach) was used. For blocks with a width of $16 \mathrm{~nm}$ (5th layer), $32 \mathrm{~nm}$ (4th layer), $64 \mathrm{~nm}$ (3rd layer), $128 \mathrm{~nm}$ (2nd layer), $256 \mathrm{~nm}$ (1st layer), the parameters of materials obtained experimentally are taken as initial conductivity data;

(2) to calculate the electrical conductivity of plane-parallel layers, including blocks with a width of $0.5 \mathrm{~nm}$ (10th layer), $1 \mathrm{~nm}$ (9th layer), $2 \mathrm{~nm}$ (8th layer), $4 \mathrm{~nm}$ (7th layer), $8 \mathrm{~nm}$ 
(6th layer), $16 \mathrm{~nm}$ (5th layer), $32 \mathrm{~nm}$ (4th layer), an effective medium model for electrical conductivity was used;

(3) to determine the electrical conductivity of plane-parallel layers, including blocks with a width of $64 \mathrm{~nm}$ (3rd layer), $128 \mathrm{~nm}$ (2nd layer), $256 \mathrm{~nm}$ (1st layer), and the composite as a whole, the method of equivalent substitution schemes was used.

Table 1. Method for determining the electrical conductivity of a layered nanocomposite with a periodic structure.

\begin{tabular}{|c|c|c|c|c|c|}
\hline $\begin{array}{c}\mathbf{n} \\
\text { Layer }\end{array}$ & $\begin{array}{l}\Delta \text { the Block Included } \\
\text { in the Layer, nm }\end{array}$ & $\begin{array}{c}\text { Quantity Blocks in a } \\
\text { Layer }\end{array}$ & $\begin{array}{c}\text { the Method of } \\
\text { Determining the } \sigma \\
\text { of the Block }\end{array}$ & $\begin{array}{c}\text { Method of } \\
\text { Determination } \\
\text { Layer } \sigma\end{array}$ & $\begin{array}{c}\text { Method for } \\
\text { Determining the } \sigma \text { of } \\
\text { the Entire } \\
\text { Nanocomposite }\end{array}$ \\
\hline 10 & 0.5 & 1024 & \multirow{6}{*}{$\begin{array}{c}\text { Kubo-Greenwood } \\
\text { equations }\end{array}$} & \multirow{7}{*}{$\begin{array}{c}\text { effective } \\
\text { environment } \\
\text { model }\end{array}$} & \multirow{10}{*}{$\begin{array}{c}\text { method } \\
\text { of equivalent circuits }\end{array}$} \\
\hline 9 & 1 & 512 & & & \\
\hline 8 & 2 & 256 & & & \\
\hline 7 & 4 & 128 & & & \\
\hline 6 & 8 & 64 & & & \\
\hline 5 & 16 & 32 & & & \\
\hline 4 & 32 & 16 & \multirow{4}{*}{$\begin{array}{l}\text { experimental } \\
\text { values }\end{array}$} & & \\
\hline 3 & 64 & 8 & & method & \\
\hline 2 & 128 & 4 & & of equivalent & \\
\hline 1 & 256 & 2 & & circuits & \\
\hline
\end{tabular}

The essence of the theory of generalized conductivity is that a particular result of studying some properties of the composite (for example, the dielectric constant) can be extended to other characteristics of the composite [15] (for example, electrical conductivity, thermal conductivity, magnetic permeability, etc.) if the equations describing the regularities of the considered processes are mathematically equivalent. The task is to find the electrical conductivity of the investigated nanocomposite by the known values of the dielectric constant and information on the structure of the nanocomposite. Maxwell's equations describing the conducting and dielectric properties of a heterogeneous system are as follows (Table 1):

Due to the equivalence of mathematical equations (Table 2), the relationship between the electrical conductivity $\sigma$ and the dielectric constant $\varepsilon$ will be determined by the relation: $\sigma=\varepsilon\left(\varepsilon_{0} i \omega\right)$, where $\varepsilon_{0}$ is the dielectric constant [16].

Table 2. Analogy between the equations describing the conductive and dielectric properties of a material (Delectric induction, $j$-current density, $\varphi$ potential).

\begin{tabular}{cc}
\hline Physical Property of the Material & Equations for a Heterogeneous System \\
\hline Electrical permittivity $\varepsilon$ & $D=-\varepsilon \times \nabla E ;$ \\
& $\operatorname{div} D=0$. \\
Electrical conductivity $\sigma$ & $\begin{array}{c}j \\
\text { div } j=0 .\end{array}$ \\
\hline
\end{tabular}

2.2. Mathematical Model of Electrical Conductivity of a Layered Nanocomposite of a Self-Similar Structure Based on the Kubo-Greenwood Equations

A quantum mechanical approach is used to find the electrical conductivity of the nanocomposite blocks included in layers 10 to 6 in.

Since the structure under study is anisotropic, the electrical conductivity of the nanocomposite will be represented by a second-rank tensor:

$$
\sigma_{i j}=\left(\begin{array}{ccc}
\sigma_{x x} & 0 & 0 \\
0 & \sigma_{y y} & 0 \\
0 & 0 & \sigma_{z z}
\end{array}\right)
$$


The nanocomposite block, which is affected by an alternating electric field, is considered $\bar{E}(t)$, under the action of which an electric current density pulse occurs in the system $\bar{J}(t)$. According to [17], $\bar{E}(t), \bar{J}(t)$ can be written as Fourier integrals:

$$
\bar{E}(t)=\int_{-\infty}^{+\infty} \bar{E}_{\omega} e^{-i \omega t} \frac{d \omega}{2 \pi}, \quad \bar{J}(t)=\int_{-\infty}^{+\infty} \bar{J}_{\omega} e^{-i \omega t} \frac{d \omega}{2 \pi}
$$

Based on Ohm's law, the complex electrical conductivity is a coefficient in a complex form $\sigma(\omega)=\sigma^{\prime}(\omega)+i \sigma^{/ /}(\omega)$ between Fourier components $\bar{E}_{\omega} u \bar{J}_{\omega}$. Accordingly:

$$
\bar{J}_{\omega}=\left(\sigma^{\prime}(\omega)+i \sigma^{/ /}(\omega)\right) \bar{E}_{\omega}
$$

The total energy absorbed by all the electrons of the elementary cells included in the nanocomposite block will be written as:

$$
E_{f u l l}=V \int_{-\infty}^{+\infty} \bar{J}(t) \bar{E}(t) d t=V \int_{-\infty}^{+\infty} \sigma^{\prime}(\omega)\left|\bar{E}_{\omega}\right|^{2} \frac{d \omega}{2 \pi}
$$

Thus, the contribution value of each Fourier component is characterized by the real part of the complex electrical conductivity.

To simulate the electrical conductivity of a nanocomposite block, we will use the KuboGreenwood approach [18-21], based on the calculation of the total energy absorbed by the electrons of the system under study, which is represented as an integral $\int_{-\infty}^{+\infty} \sigma^{\prime}(\omega)\left|\bar{E}_{\omega}\right|^{2} \frac{d \omega}{2 \pi}$, from the coefficients of which it will be possible to find the real part of the complex electrical conductivity.

In the general case, a system of stationary equations is considered, which are determined by the energies of electrons $\epsilon_{n}, \epsilon_{m}$ normal and excited states, respectively. The corresponding wave functions are assigned to the electron energies $\Psi_{n}$ and $\Psi_{m}$. A disturbing operator acts on the system $\hat{V}_{p}$. Since an anisotropic medium is considered, it is necessary to take into account the directions of the electric field along each axis. The perturbing operator along each axis will have the form:

$$
\hat{V}_{p}=e \bar{E}(t) \hat{x}, \quad \hat{V}_{p}=e \bar{E}(t) \hat{y}, \quad \hat{V}_{p}=e \bar{E}(t) \hat{z} .
$$

The total probability of transition from level $\mathrm{n}$ to level $\mathrm{m}$ will be equal to:

$$
\begin{aligned}
& P_{(n \rightarrow m)_{x}}=\frac{e^{2}}{\hbar^{2}}\left\langle\Psi_{n}|\hat{x}| \Psi_{m}\right\rangle^{2}\left|E_{\frac{\epsilon_{m}-\epsilon_{n}}{\hbar}}\right|^{2} \\
& P_{(n \rightarrow m)_{y}}=\frac{e^{2}}{\hbar^{2}}\left\langle\Psi_{n}|\hat{y}| \Psi_{m}\right\rangle^{2}\left|E_{\frac{\epsilon_{m}-\epsilon_{n}}{\hbar}}\right|^{2} \\
& P_{(n \rightarrow m)_{z}}=\frac{e^{2}}{\hbar^{2}}\left\langle\Psi_{n}|\hat{z}| \Psi_{m}\right\rangle^{2}\left|E_{\frac{\epsilon_{m}-\epsilon_{n}}{\hbar}}\right|^{2}
\end{aligned}
$$

When moving from one level to another, the total energy of the system increases by:

$$
\begin{aligned}
& E_{(n \rightarrow m)_{x}}^{f u l l}=\frac{e^{2}}{\hbar^{2}}\left(\epsilon_{m}-\epsilon_{n}\right)\left\langle\Psi_{n}|\hat{x}| \Psi_{m}\right\rangle^{2}\left|E_{\frac{\epsilon_{m}-\epsilon_{n}}{\hbar}}\right|^{2} \\
& E_{(n \rightarrow m)_{y}}^{f u l l}=\frac{e^{2}}{\hbar^{2}}\left(\epsilon_{m}-\epsilon_{n}\right)\left\langle\Psi_{n}|\hat{y}| \Psi_{m}\right\rangle^{2}\left|E_{\frac{\epsilon_{m}-\epsilon_{n}}{\hbar}}\right|^{2} \\
& E_{(n \rightarrow m)_{z}}^{f u l l}=\frac{e^{2}}{\hbar^{2}}\left(\epsilon_{m}-\epsilon_{n}\right)\left\langle\Psi_{n}|\hat{z}| \Psi_{m}\right\rangle^{2}\left|E_{\frac{\epsilon_{m}-\epsilon_{n}}{\hbar}}\right|^{2}
\end{aligned}
$$


In statistical analysis, the expression (7) must be multiplied by the fill numbers $f\left(\epsilon_{n}\right)$, $f\left(\epsilon_{m}\right)$, determined by the Fermi distribution for the normal and excited states, respectively. The total energy absorbed by the system will have the form:

$$
\begin{aligned}
& E_{x}^{f u l l}=\frac{e^{2}}{2 \hbar^{2}} \sum_{n m}\left(\epsilon_{m}-\epsilon_{n}\right)\left\langle\Psi_{n}|\hat{x}| \Psi_{m}\right\rangle^{2}\left|E_{\frac{\epsilon_{m}-\epsilon_{n}}{\hbar}}\right|^{2}\left(f\left(\epsilon_{m}\right)-f\left(\epsilon_{n}\right)\right) \\
& E_{y}^{f u l l}=\frac{e^{2}}{2 \hbar^{2}} \sum_{n m}\left(\epsilon_{m}-\epsilon_{n}\right)\left\langle\Psi_{n}|\hat{y}| \Psi_{m}\right\rangle^{2}\left|E_{\frac{\epsilon_{m}-\epsilon_{n}}{\hbar}}\right|^{2}\left(f\left(\epsilon_{m}\right)-f\left(\epsilon_{n}\right)\right) \\
& E_{z}^{f u l l}=\frac{e^{2}}{2 \hbar^{2}} \sum_{n m}\left(\epsilon_{m}-\epsilon_{n}\right)\left\langle\Psi_{n}|\hat{z}| \Psi_{m}\right\rangle^{2}\left|E_{\frac{\epsilon_{m}-\epsilon_{n}}{\hbar}}\right|^{2}\left(f\left(\epsilon_{m}\right)-f\left(\epsilon_{n}\right)\right)
\end{aligned}
$$

In order to represent expressions (8) in integral form, it is necessary to use the $\delta$ -function. We get:

$$
\begin{aligned}
& E_{x}^{f u l l}=\int \frac{e^{2}}{2 \hbar^{2}} \sum_{n m}\left(\epsilon_{m}-\epsilon_{n}\right)\left\langle\Psi_{n}|\hat{x}| \Psi_{m}\right\rangle^{2}\left|E_{\omega}\right|^{2}\left(f\left(\epsilon_{m}\right)-f\left(\epsilon_{n}\right)\right) \delta\left(\omega-\frac{\epsilon_{m}-\epsilon_{n}}{\hbar}\right) d \omega \\
& E_{y}^{f u l l}=\int \frac{e^{2}}{2 \hbar^{2}} \sum_{n m}\left(\epsilon_{m}-\epsilon_{n}\right)\left\langle\Psi_{n}|\hat{y}| \Psi_{m}\right\rangle^{2}\left|E_{\omega}\right|^{2}\left(f\left(\epsilon_{m}\right)-f\left(\epsilon_{n}\right)\right) \delta\left(\omega-\frac{\epsilon_{m}-\epsilon_{n}}{\hbar}\right) d \omega \\
& E_{z}^{f u l l}=\int \frac{e^{2}}{2 \hbar^{2}} \sum_{n m}\left(\epsilon_{m}-\epsilon_{n}\right)\left\langle\Psi_{n}|\hat{z}| \Psi_{m}\right\rangle^{2}\left|E_{\omega}\right|^{2}\left(f\left(\epsilon_{m}\right)-f\left(\epsilon_{n}\right)\right) \delta\left(\omega-\frac{\epsilon_{m}-\epsilon_{n}}{\hbar}\right) d \omega
\end{aligned}
$$

To obtain an expression for electrical conductivity, we substitute (9) into (7) and integrate the equations. The integration is carried out by the Simpson method, with a step of $0.1 \mathrm{~nm}$. As a result, we obtain modified Kubo-Greenwood equations describing the electrical conductivity of the nanocomposite block and taking into account the anisotropy of electrical conductivity and electron spin:

$$
\begin{aligned}
& \sigma_{x x}=\frac{2 \pi e^{2} \hbar^{3}}{m_{e}^{2} V}\left(\frac{\partial \Psi_{n}}{\partial x}+\frac{\partial \Psi_{m}}{\partial x}\right)^{2} \frac{f\left(\epsilon_{m}\right)-f\left(\epsilon_{n}\right)}{\epsilon_{m}-\epsilon_{n}} \delta\left(\epsilon_{m}-\epsilon_{n}-\hbar \omega\right) ; \\
& \sigma_{y y}=\frac{2 \pi e^{2} \hbar^{3}}{m_{e}^{2} V}\left(\frac{\partial \Psi_{n}}{\partial y}+\frac{\partial \Psi_{m}}{\partial y}\right)^{2} \frac{f\left(\epsilon_{m}\right)-f\left(\epsilon_{n}\right)}{\epsilon_{m}-\epsilon_{n}} \delta\left(\epsilon_{m}-\epsilon_{n}-\hbar \omega\right) \\
& \sigma_{z z}=\frac{2 \pi e^{2} \hbar^{3}}{m_{e}^{2} V}\left(\frac{\partial \Psi_{n}}{\partial z}+\frac{\partial \Psi_{m}}{\partial z}\right)^{2} \frac{f\left(\epsilon_{m}\right)-f\left(\epsilon_{n}\right)}{\epsilon_{m}-\epsilon_{n}} \delta\left(\epsilon_{m}-\epsilon_{n}-\hbar \omega\right),
\end{aligned}
$$

where $\epsilon_{n}, \epsilon_{m}$-the electron energies of the normal and excited states, respectively; $f\left(\epsilon_{n}\right)$, $f\left(\epsilon_{m}\right)$ - the fill numbers determined by the Fermi distribution for the normal and excited states, respectively.

The model assumes the presence of information about the energy of electrons in the normal and excited states $\left(\epsilon_{n}, \epsilon_{m}\right)$, and also about the filling numbers $f\left(\epsilon_{n}\right), f\left(\epsilon_{m}\right)$, determined by the Fermi distribution for the normal and excited states. To obtain them, the method of electronic minimization is used. Differentiation is performed by a four-point method with a step of $0.1 \mathrm{~nm}$. Values of wave functions $\Psi_{n} \Psi_{m}$ and the other parameters included in Equation (10) are taken the same as in the Agranovich-Ginzburg approach used to calculate the permittivity [21-27].

For the blocks included in layers 1 to 5 , in accordance with the above, the parameters of materials obtained experimentally are taken as the initial data of electrical conductivity.

\section{Algorithm for Reconstructing the Electrical Conductivity Tensor from the Values of the Electric Field Potential}

In a composite medium $a \times c$ (Figure 1) with anisotropic electrical properties, the following coefficient inverse charge transfer problem by determining the electrical conductivity tensor is considered. 


$$
\begin{gathered}
\sigma=\left[\begin{array}{ll}
\sigma_{11}(\varphi) & \sigma_{12}(\varphi) \\
\sigma_{21}(\varphi) & \sigma_{21}(\varphi)
\end{array}\right] \\
\frac{\partial}{\partial x}\left(\sigma_{11}(\varphi) \frac{\partial \varphi}{\partial x}\right)+\frac{\partial}{\partial x}\left(\sigma_{12}(\varphi) \frac{\partial \varphi}{\partial x}\right)+\frac{\partial}{\partial y}\left(\sigma_{21}(\varphi) \frac{\partial \varphi}{\partial x}\right)+\frac{\partial}{\partial y}\left(\sigma_{22}(\varphi) \frac{\partial \varphi}{\partial x}\right)=e \mathrm{C} \rho \frac{\partial \varphi}{\partial t}, \\
x \in(0 ; a), y \in(0 ; c), t>0, \\
\varphi(x, 0, t)=\varphi(x, c, t)=\varphi(0, y, t)=\varphi(a, y, t)=\left.\varphi\right|_{S} \\
\varphi(x, y, 0)=\varphi_{0}
\end{gathered}
$$

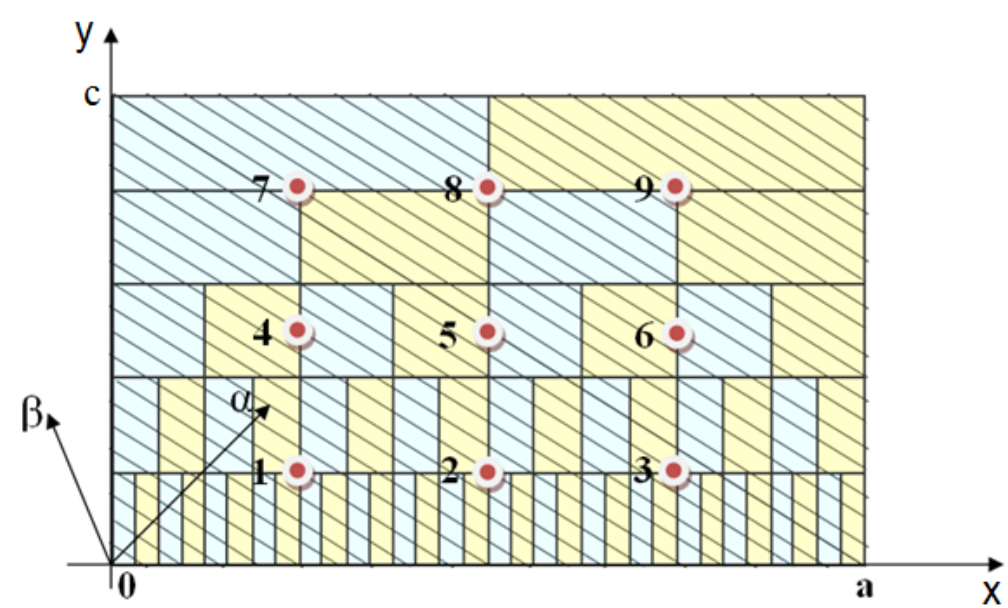

Figure 1. Computational domain of a nanocomposite medium.

The boundary conditions are taken as the maximum value of the field potential $\left.\varphi\right|_{S}=\varphi_{\max }$, and the initial one is taken as the minimum $\left.\varphi\right|_{0}=\varphi_{\min }$, i.e., $\varphi_{\min } \leq \varphi \leq \varphi_{\max }$. To close the coefficient inverse problem of charge transfer in an anisotropic composite medium, the values of the electric field strength at nine points are set as a function of time $\varphi\left((x, y)_{\xi}, t_{\eta}\right)=\widetilde{\varphi}_{\xi, \eta}, \xi=\overline{1,9}, \eta=\overline{1, H}$. The values of the electric field strength are calculated based on the data on the dielectric constant of the nanocomposite obtained.

A two-dimensional case is considered; therefore, the number of points with the values of the electric field strength in the direction of each coordinate axis should be at least two; and, since the composite medium is anisotropic, then, in accordance with the spatial template for finite-difference schemes, the number of these points in the coordinate directions should be at least three. Thus, the minimum possible number of spatial points is assumed, equal to nine, with field strength values depending on time [28].

The nonlinear components of the electrical conductivity tensor are determined through the principal coefficients $\sigma_{\alpha}, \sigma_{\beta}$ and the orientation angle $\varphi$ of the principal axes $O \alpha$ and $O \beta$ as follows:

$$
\begin{gathered}
\sigma_{11}(\varphi)=\sigma_{\alpha}(\varphi) \cos ^{2} \varphi+\sigma_{\beta}(\varphi) \sin ^{2} \varphi \\
\sigma_{22}(\varphi)=\sigma_{\alpha}(\varphi) \sin ^{2} \varphi+\sigma_{\beta}(\varphi) \cos ^{2} \varphi \\
\left.\sigma_{12}(\varphi)=\sigma_{21}(\varphi)=\sigma_{\alpha}(\varphi)-\sigma_{\beta}(\varphi)\right) \sin \varphi \cos \varphi
\end{gathered}
$$

To solve the problem, the method of parametric identification is used, in which the sought functions $\sigma_{11}(\varphi), \sigma_{12}(E \varphi)=\sigma_{21}(\varphi), \sigma_{22}(\varphi)$ are found in the form of a linear combination of basis functions $G_{n}(E)$ defined on finite elements as finite segments $\Delta \varphi_{n}, n=\overline{1, N-1}\left(\varphi_{\min } \leq \varphi \leq \varphi_{\max }\right)$, and these basis functions are assigned to each node:

$$
\varphi_{n}=\varphi_{\min }+\sum_{a=1}^{n} \Delta \varphi_{a-1}\left(\Delta \varphi_{0}=0\right)
$$


$n=\overline{1, N}$ and orthogonal on the segment $\varphi \in\left[\varphi_{\min }, \varphi_{\max }\right]$. The following linear-continuous basis functions are used:

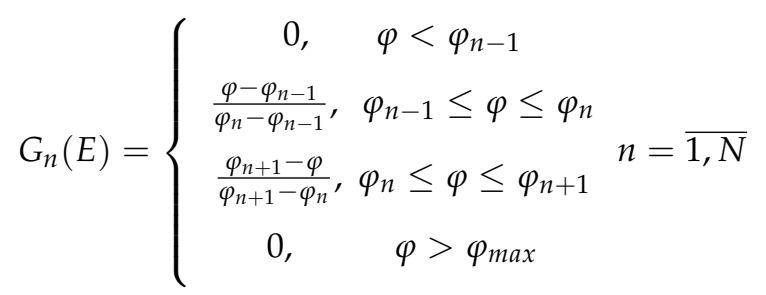

The field-dependent nonlinear components of the electrical conductivity tensor are represented in the parametric identification method as linear combinations of basis functions $G_{n}(\varphi)$ :

$$
\begin{aligned}
& \sigma_{11}(\varphi) \approx \sum_{n=1}^{N} \sigma_{11}^{n} G_{n}(\varphi), \\
& \sigma_{22}(\varphi) \approx \sum_{n=1}^{N} \sigma_{22}^{n} G_{n}(\varphi), \\
& \sigma_{12}(\varphi) \approx \sum_{n=1}^{N} \sigma_{12}^{n} G_{n}(\varphi),
\end{aligned}
$$

where the coefficients of the linear combinations $\sigma_{11}^{n}, \sigma_{22}^{n}, \sigma_{12}^{n}$ at each n-th finite element $n=\overline{1, N-1}$ are the required quantities. Based on the maximum principle, it can be argued that the electric field strength inside the region will satisfy the inequality $\varphi_{\min } \leq \varphi(x, y, t) \leq \varphi_{\max }$.

By designating $\sigma_{11}^{m}=\sigma_{11}\left(\varphi_{\min }\right), \sigma_{12}^{m}=\sigma_{12}\left(\varphi_{\min }\right), \sigma_{21}^{m}=\sigma_{21}\left(\varphi_{\min }\right), \sigma_{22}^{m}=\sigma_{22}\left(\varphi_{\min }\right)$, $\sigma_{11}^{M}=\sigma_{11}\left(\varphi_{\max }\right), \sigma_{12}^{M}=\sigma_{12}\left(\varphi_{\max }\right), \sigma_{21}^{M}=\sigma_{21}\left(\varphi_{\max }\right), \sigma_{22}^{M}=\sigma_{22}\left(\varphi_{\max }\right)$, to determine the constant components of the tensor

$$
\sigma=\left[\begin{array}{cccccc}
\sigma_{11}^{m} & \ldots & \sigma_{11}^{M} & \sigma_{12}^{m} & \ldots & \sigma_{12}^{M} \\
\sigma_{21}^{m} & \ldots & \sigma_{21}^{M} & \sigma_{22}^{m} & \ldots & \sigma_{22}^{M}
\end{array}\right]
$$

into expressions (12), the quadratic functional is introduced:

$$
S(\sigma)=\frac{1}{2} \sum_{\xi=1}^{9} \sum_{\eta=1}^{H}\left[E_{\xi, \eta}(\sigma)-\widetilde{E}_{\xi, \eta}\right]^{2}
$$

To minimize the functional, an implicit gradient descent method is used:

$$
\sigma^{(n+1)}=\sigma^{(n)}-l_{n} \operatorname{grad} S\left(\sigma^{n+1}\right)
$$

where $\mathrm{n}$ is the iteration number; $\delta_{n}$-parametric steps $\left(\delta_{n}>0\right)$.

$$
S\left(l^{(n+1)}\right)<S\left(\sigma^{(n)}\right)
$$

The initial values $l_{0}$, based on condition (19), can be chosen arbitrarily. If, as a result of iteration, condition (19) is not met, then $l_{n}$ decreases at this iteration, and the calculation is repeated on it; otherwise, when (19) is fulfilled for the next iteration, $l_{n}$ increases. The end of the iterative process is set close to zero grad $S\left(\sigma^{n+1}\right)$, i.e., under the condition $\mid$ grad $S\left(\sigma^{n+1}\right) \mid \leq \mu$, where $\mu$ is the specified accuracy.

Thus, this article proposes an algorithm for the numerical solution of the inverse coefficient problem of charge transfer in an anisotropic composite medium; the input data can be changed in accordance with a physical experiment. 


\section{Mathematical Model of Nanocomposite Electrical Conductivity Based on the Theory of Effective Medium}

The modeling of the electrical conductivity of layers from the 4 th to the 10th, including blocks with sizes from 0.5 to $32 \mathrm{~nm}$, was carried out. For this purpose, an effective environment model is used. The $\mathrm{TiO}_{2}-\mathrm{Al}_{2} \mathrm{O}_{3}$ composite is considered as an example, with the material $\mathrm{TiO}_{2}$ denoted in blue, and $\mathrm{Al}_{2} \mathrm{O}_{3}$ in yellow. Such materials are used in photochemical batteries-Gratzel cells and are of great interest for research [18]. This approach of modeling the electrical conductivity of anisotropic composites was used in [20]; however, the author considered composites with cylindrical and spherical inclusions.

In this paper, based on this approach, modeling of a layered nanocomposite with a periodic structure is carried out (Figure 2). Since an anisotropic medium is considered in this paper, the complex electrical conductivity will be represented by a tensor:

$$
\sigma_{e f f_{i j}}=\left(\begin{array}{ccc}
\sigma_{e f f_{x x}} & 0 & 0 \\
0 & \sigma_{e f f_{y y}} & 0 \\
0 & 0 & \sigma_{e f f_{z z}}
\end{array}\right)=f\left(\sigma_{i j_{1}}, \sigma_{i j_{2}}, \triangle\right)
$$

where $\sigma_{i j_{1}}, \sigma_{i j_{2}}$-the electrical conductivity tensor of blocks of the 1st and 2nd type of material, respectively. The effective conductivity is found from the basic macroscopic equations $\operatorname{rot} E=0$, divj $=0$ and Ohm's law: $j=\sigma E$.

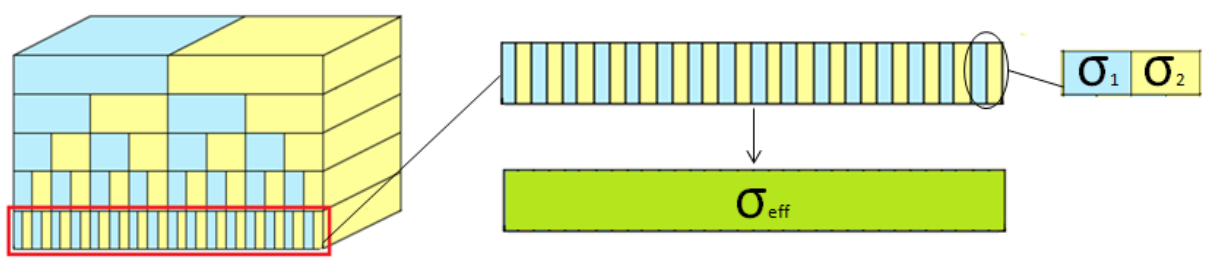

Figure 2. Schematic representation of an effective environment.

Let's average the vector $\mathrm{J}$ over the volume $\mathrm{V}$ of the object of study $J=j-\sigma E$. For a nanocomposite consisting of $\mathrm{n}$ layers

$$
\langle j-\sigma E\rangle=-\sum_{i}^{n}\left(\sigma-\sigma_{i}\right) \frac{1}{V} \int_{V_{i}}^{\sigma E} E d V
$$

where $\sigma_{i}$-the conduction tensor of the block, and the integral is taken by its volume $V_{i}$. According to Maxwell 's equations, the average value is $J=0$, accordingly, the left side of the equation is also zero. Thus, expression (21) takes the form:

$$
\sum_{i=1}^{n} N_{i}\left(\sigma-\sigma_{i}\right)\left\langle\int_{V_{i}}^{\sigma} E d V\right\rangle_{i}=0
$$

where $N_{i}$-the number of blocks in the layer, integration is carried out by the volume of the block. By $v_{i}$ the averaging of the i-th component is implied.

The approximation of the effective medium model consists in replacing the average value $\left\langle\int_{V_{i}}^{\sigma} E d V\right\rangle_{i}$ on the integral of the electric field strength $E_{i}$ inside a separate i-th block placed in a layer with a conductivity tensor $\sigma_{\text {eff }}$. Thus, the basic equation of the effective medium model will have the following form:

$$
\sum_{i=1}^{n} v_{i}\left(\sigma_{e f f}-\sigma_{i}\right)\langle E\rangle_{i}=0
$$


where $v_{i}$-dimensionless concentration of the i-th component $\left(\sum_{i=1}^{n} v_{i}=1\right)$. For a layered nanocomposite comprising blocks of two types of materials $v_{i}=\triangle_{1}+\triangle_{2}$. $\langle E\rangle_{i}$ - the average value of the electric field strength.

Models for the layered structure corresponding to the components $\sigma_{\mathrm{eff}_{\mathrm{xx}}}, \sigma_{\mathrm{eff}_{\mathrm{yy}}}$, have the form:

$$
\begin{aligned}
& J=\left(\triangle_{1} \sigma_{x x_{1}}+\triangle_{2} \sigma_{x x_{2}}\right) E \\
& \sigma_{e f f_{x x u}}=\triangle_{1} \sigma_{x x_{1}}+\triangle_{2} \sigma_{x x_{2}}
\end{aligned}
$$

For components $\sigma_{e f f_{z z}}$ :

$$
\begin{gathered}
E=J\left(\frac{\triangle_{1}}{\sigma_{z z_{1}}}+\frac{\triangle_{2}}{\sigma_{z z_{2}}}\right) \\
\sigma_{e f f_{z z}}=\frac{\sigma_{z z_{1}} \sigma_{z z_{2}}}{\triangle_{1} \sigma_{z z_{1}}+\triangle_{2} \sigma_{z z_{2}}}
\end{gathered}
$$

Next, to calculate the components of the electrical conductivity tensor of layers 1 through 3 and the composite as a whole, we use the method of equivalent circuits.

Considering that $\sigma=\frac{1}{Z}$, using the equivalent scheme, we obtain:

$$
\begin{aligned}
& \sigma_{x x, y y}^{\prime}=\frac{\left[\sigma_{1}^{\prime} d_{1}\left(\sigma_{2}^{/ 2}+\sigma_{2}^{/ / 2}\right)+\sigma_{2}^{/ /} d_{2}\left(\sigma_{1}^{/ 2}+\sigma_{1}^{/ / 2}\right)\right]\left(\mathrm{d}_{1}+\mathrm{d}_{2}\right)}{\left(\sigma_{2}^{\prime} \mathrm{d}_{1}+\sigma_{1}^{\prime} \mathrm{d}_{1}\right)^{2}+\left(\sigma_{2}^{\prime /} \mathrm{d}_{2}+\sigma_{1}^{/ /} \mathrm{d}_{2}\right)^{2}} ; \sigma_{\mathrm{zz}}^{\prime}=\frac{\left(\sigma_{1}^{\prime} \mathrm{d}_{1}+\sigma_{2}^{\prime} \mathrm{d}_{2}\right)}{\left(\mathrm{d}_{1}+\mathrm{d}_{2}\right)} ; \\
& \sigma_{\mathrm{xx}, \mathrm{yy}}^{\prime \prime}=\frac{\left[\sigma_{1}^{\prime /} \mathrm{d}_{1}\left(\sigma_{2}^{/ 2}+\sigma_{2}^{/ / 2}\right)+\sigma_{2}^{/ /} \mathrm{d}_{2}\left(\sigma_{1}^{/ 2}+\sigma_{1}^{/ / 2}\right)\right]\left(\mathrm{d}_{1}+\mathrm{d}_{2}\right)}{\left(\sigma_{2}^{\prime} \mathrm{d}_{1}+\sigma_{1}^{\prime} \mathrm{d}_{2}\right)^{2}+\left(\sigma_{2}^{/ /} \mathrm{d}_{1}+\sigma_{1}^{/ /} \mathrm{d}_{2}\right)^{2}} ; \sigma_{\mathrm{zz}}^{/ /}=\frac{\left(\sigma_{1}^{\prime /} \mathrm{d}_{1}+\sigma_{2}^{/ /} \mathrm{d}_{2}\right)}{\left(\mathrm{d}_{1}+\mathrm{d}_{2}\right)}
\end{aligned}
$$

\section{Result}

The dependences of conductivity on the electric field strength are modeled by the method proposed in the work and using the theory of generalized conductivity. The results obtained are shown in Figure 3. We wanted to show the effect of a small magnitude of the electric field voltage. This is due to the study of fundamental physical phenomena, in particular, the study of the electrocaloric (EC) effect in nanocomposites with a periodic structure [28]. It is the strength of a small electric field that makes it possible to detect such effects in periodic structures. At high electric field strengths, this effect can be neglected.
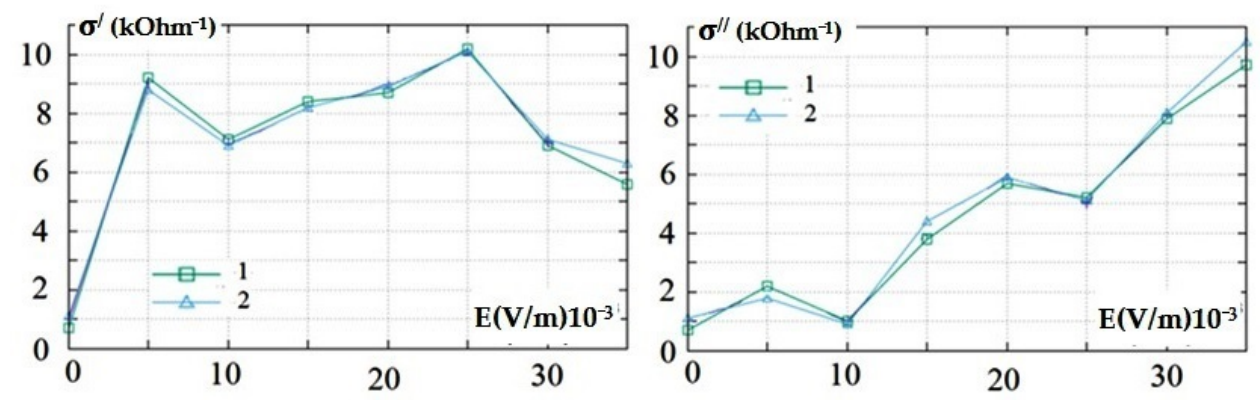

Figure 3. Dependence of the complex electrical conductivity on the field strength.

A simulation of a nanocomposite with a periodic structure is carried out, the equivalent scheme of which has the form (Figure 4). 


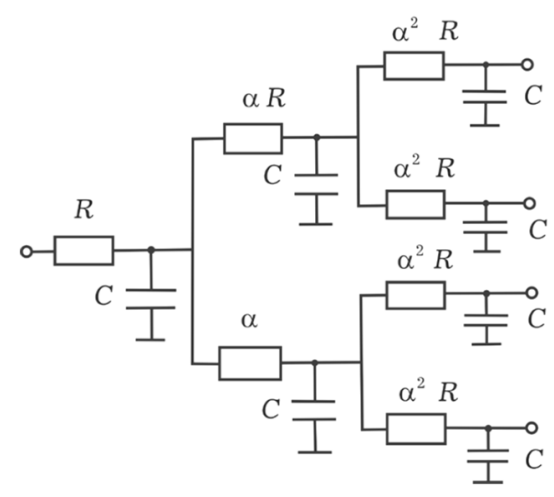

Figure 4. Equivalent nanocomposite scheme.

The impedance of the circuit is described in [22] and depends on the parameters of the equivalent electrical circuit, fragmentation and fractality. A software application has been developed that allows modeling the frequency dependences of the impedance, the simulation results are presented on Figure 5.

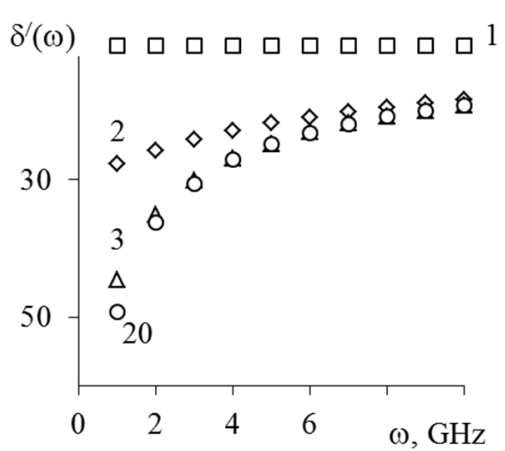

(a)

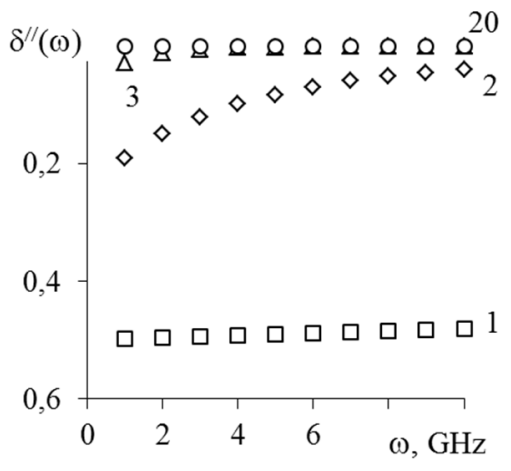

(b)

Figure 5. The effect of the fractal parameter on the frequency dependence of the impedance: (a) real and (b) imaginary components.

The qualitative change in the impedance dependence at different frequencies indicates that the system under study is self-similar. The self-similarity of the properties of an object at different scales indicates that a composite material, the equivalent scheme of which has a hierarchical structure, can be considered as a fractal formation.

To study the electrical conductivity of layered nanocomposites with a periodic structure, a number of computational experiments were carried out for the first time, as a result of which the following properties and regularities were established. It was found that a layered nanocomposite with a periodic structure has electrical properties that differ significantly from the properties of homogeneous materials. The fractality level of the nanocomposite and its geometric configuration significantly affect the electrical conductivity. A shift of the curves is observed in the regions of electronic and ionic polarizations, which is presumably associated with relaxation losses.

The scientific significance is determined by the fact that the mathematical models and methods proposed in this work make it possible to study fundamental physical phenomena occurring in layered nanocomposites with a periodic structure in an external electromagnetic field.

The practical significance is due to the results obtained in the course of the study, which open up the possibility of using layered nanocomposites with a periodic structure in modern absorbing and reflecting devices. In particular, the nonlinear nature of electrical conductivity in such a nanocomposite medium was discovered, and the possibility of con- 
trolling resonance bursts, which would allow the design of materials with predetermined electrical properties.

\section{Conclusions}

The presented method of modeling the electrical conductivity based on the KuboGreenwood equations effective environment model method of equivalent circuits use will allow making predictions to describe the electrical conductivity of the nanocomposite under study. This can be useful when creating smart materials with specified electrophysical properties, thyristors, absorbing screens, and elements of radar devices.

Based on the results obtained, it can be seen that the dependences obtained using the method proposed in this article are in good agreement with the results obtained using the theory of generalized conductivity. The advantage of the proposed method is the removal of restrictions imposed on the theory of generalized conductivity associated with the need to set the permittivity.

Author Contributions: Conceptualization, E.P.; Data curation, E.P., I.A. and K.B.; Formal analysis, V.D. and K.B.; Investigation, K.B.; Methodology, E.P., V.D. and D.S.; Project administration, S.K.; Resources, S.K.; Software, S.K. and D.S.; Supervision, D.S.; Validation, I.A., V.D. and E.D.; Visualization, I.A. and E.D.; Writing—original draft, E.D. All authors have read and agreed to the published version of the manuscript.

Funding: This research received no external funding.

Institutional Review Board Statement: Not applicable.

Informed Consent Statement: Not applicable.

Conflicts of Interest: The authors declare no conflict of interest.

\section{References}

1. Bunde, A.; Dieterich, W. Percolation in composites. J. Electroceramics 2000, 5, 81-92. [CrossRef]

2. Shklovskiı̆, B.I.; Efros, A.L. Percolation theory and conductivity of strongly inhomogeneous media. Sov. Phys. Uspekhi 1975, 18, 845 . [CrossRef]

3. Khasanshin, I. Application of an Artificial Neural Network to Automate the Measurement of Kinematic Characteristics of Punches in Boxing. Appl. Sci. 2021, 11, 1223. [CrossRef]

4. Korchagin, S.; Serdechny, D.; Kim, R.; Terin, D.; Bey, M. The use of machine learning methods in the diagnosis of diseases of crops. E3S Web Conf. 2020, 176, 04011. [CrossRef]

5. Qiao, R.; Brinson, L.C. Simulation of interphase percolation and gradients in polymer nanocomposites. Compos. Sci. Technol. 2009, 69, 491-499. [CrossRef]

6. Babaev, A.A.; Khokhlachev, P.P.; Nikolaev, Y.A.; Terukov, E.I. Temperature Dependence of Resistivity and Current-Voltage Characteristics of the Films of Composites Based on Modified Carbon Multiwalled Nanotubes and Graphite. Ph.D. Thesis, Sumy State University, Sumy, Ukraine, 2013.

7. Perov, G.V. Modeling of Ionic Conductivity of a Dielectric with an Inhomogeneous Blocking Surface of Micro and Nanomatrix Components; Salman Bulletin of SibGUTI: Moscow, Russia, 2008; pp. 25-30.

8. Edvabnik, V.G. On the Theory of Generalized Conductivity of Mixtures; Modern Problems of Science and Education: Moscow, Russia, 2015; pp. 76-129.

9. Lukomskaya, A.I.; Badenkov, P.F.; Kepersha, L.M. Thermal Bases of Vulcanization of Rubber Products; Chemistry: Moscow, Russia, 1972; 359p.

10. Moiseev, G. Forecasting oil tanker shipping market in crisis periods: Exponential smoothing model application. Asian J. Shipp. Logist. 2021, 37, 239-244. [CrossRef]

11. Korchagin, S.A.; Terin, D.V.; Klinaev, Y.V.; Romanchuk, S.P. Simulation of Current-Voltage Characteristics of Conglomerate of Nonlinear Semiconductor Nanocomposites. In Proceedings of the 2018 International Conference on Actual Problems of Electron Devices Engineering (APEDE), Saratov, Russia, 27-28 September 2018; pp. 397-399.

12. Knyazev, D.V. Ab inito calculation of transport and optical properties of aluminum: Influence of simulation parameters. Levashov Comput. Mater. Sci. 2013, 79, 817-829. [CrossRef]

13. Shirokanev, A.S.; Andriyanov, N.A.; Ilyasova, N.Y. Development of vector algorithm using CUDA technology for threedimensional retinal laser coagulation process modeling. Comput. Opt. 2021, 45, 427-437. [CrossRef]

14. Soloviev, V. Fintech Ecosystem in Russia. In Proceedings of the 2018 Eleventh International Conference "Management of Large-Scale System Development" (MLSD 2018), Moscow, Russia, 1-3 October 2018. [CrossRef] 
15. Sebyakin, A.; Soloviev, V.; Zolotaryuk, A. Lecture Notes in Computer Science (including subseries Lecture Notes in Artificial Intelligence and Lecture Notes in Bioinformatics) ToM 12645 LNCS. In Proceedings of the16th International Conference on Diversity, Divergence, Dialogue, iConference 2021, Beijing, China, 17-31 March 2021. [CrossRef]

16. Gadomski, A.; Kruszewska, N.; Rubi, J.M. Derivation of the refractive index of lipid monolayers at an air-water interface. Opt. Mater. 2019, 93, 1-5. [CrossRef]

17. Soloviev, V.; Titov, N.; Smirnova, E. Coking Coal Railway Transportation Forecasting Using Ensembles of ElasticNet, LightGBM, and Facebook Prophet. In Proceedings of the International Conference on Machine Learning, Optimization, and Data Science; Springer: Cham, Switzerland, 2020; pp. 181-190.

18. Palko, N.; Potemkin, V.; Grishina, M. Theoretical study of the surface structure of anatase nanoparticles: Effect on dye adsorption and photovoltaic properties. N. J. Chem. 2020, 44, 17267-17276. [CrossRef]

19. Kazarov, B.A.; Mityugova, O.A.; Altukhov, V.I. Modeling of properties of wide-band nanostructured materials within the framework of methods of dynamic Green functions and formulas of the Kubo-Greenwood type. Fundam. Res. 2015, 3, 76-84.

20. Abrahams, E.; Anderson, P.W.; Licciardello, D.C.; Ramakrishnan, T.V. Scaling Theory of Localization: Absence of Quantum Diffusion in Two Dimensions. Phys. Rev. Lett. 1979, 42, 673-687. [CrossRef]

21. Balagurov, B.Y. Conductivity of a three-dimensional composite model with structural anisotropy. JETF 2016, 150, 401-410.

22. Balkhanov, V.K. Modeling of a fractal medium of the Kantorov type by a hierarchical equivalent electrical circuit. Phys. Wave Process. Radio Eng. Syst. 2008, 11, 26-30.

23. Yerznkyan, B.; Bychkova, S.; Gataullin, T.; Gataullin, S. The Sufficiency Principle as the Ideas Quintessence of the Club of Rome. Montenegrin J. Econ. 2019, 15, 21-29. [CrossRef]

24. Xu, S.; Zhang, Y.; Cho, J.; Lee, J.; Huang, X. Stretchable batteries with self-similar serpentine interconnects and integrated wireless recharging systems. Nat. Commun. 2013, 4, 1543. [CrossRef] [PubMed]

25. Zeng, Q.H.; Yu, A.B.; Lu, G.Q. Multiscale modeling and simulation of polymer nanocomposites. Prog. Polym. Sci. 2020, 33, 191-269. [CrossRef]

26. Belhimria, R.; Boukheir, S.; Samir, Z.; Len, A.; Szakál, A. El Hasnaoui Polyester/Graphite Percolating Composite: Structural and Dielectric Analyses. J. Electron. Mater. 2021, 50, 6920-6928. [CrossRef]

27. Zuo, Y.T.; Liu, H.J. Fractal approach to mechanical and electrical properties of graphene/sic composites. Facta Univ. Ser. Mech. Eng. 2021, 19, 271-284. [CrossRef]

28. Starkov, A.S.; Karmanenko, S.F.; Pakhomov, O.V.; Eskov, A.V.; Semikin, D.; Hagberg, I. Electrocaloric response of a ferroelectric to the action of a periodic electric field. Solid State Phys. 2009, 51, 1422-1426. [CrossRef] 